Pacific Journal of Mathematics

GENERALIZED CONVEXITY AND SURFACES OF NEGATIVE 


\title{
GENERALIZED CONVEXITY AND SURFACES \\ OF NEGATIVE CURVATURE
}

\author{
Paul A. Clement
}

Introduction. In a study [4] of surfaces whose Gaussian, or total, curvature $K$ satisfies the relation $K \leq 0$, a number of functions having geometrical significance have been shown to be convex. In the present paper, a study of surfaces whose Gaussian curvature satisfies $K \leq K_{0}$, where $K_{0}$ is a negative constant, leads to the determination of a class of functions which are subfunctions (defined in $\S 1.1$ ) of a two-parameter family of functions determined by the bound $K_{0}$. This is a natural generalization because the convexity property is equivalent to the subfunction property with respect to the particular two-parameter family (nonvertical straight lines) determined by the bound $K_{0}=0$.

A main objective will be to exhibit functions which have a geometrical significance and also have the subfunction property for surfaces with $K \leq K_{0}$. This property then implies certain inequality relations for functions associated with certain geometrical configurations on such surfaces.

\section{SubFunctions}

1.1. Definitions. A real-valued function $g(x)$ of a single real variable $x$ defined on an open interval $(a, b)$, with $-\infty \leq a<x<b \leq+\infty$, is said to be a convex function of $x$ provided $g(x)$ satisfies the inequality

$$
g\left[t x_{1}+(1-t) x_{2}\right] \leq \operatorname{tg}\left(x_{1}\right)+(1-t) g\left(x_{2}\right)
$$

for all $x_{1}, x_{2}$ in $(a, b)$ and for all $t$ on the range $0 \leq t \leq 1$. If $g(x)$ is of class $C^{2}$, it is convex if and only if $g^{\prime \prime}(x) \geq 0$ throughout the interval.

Geometrically, (1.11) indicates that no part of the graph of the curve $y=g(x)$ lies above the chord joining two points upon it within the interval $(a, b)$.

A generalization of the foregoing characteristic geometric property of convex

Received July 6, 1952. This paper is largely a condensation of a June, 1949, doctoral dissertation, University of California, Los Angeles; I gratefully acknowledge the guidance of Professor Beckenbach.

Pacific J. Math. 3 (1953), 333-368 
functions leads to the theory of subfunctions [3]. Let $\left\{h_{\alpha \beta}(x)\right\}$ be a twoparameter family of continuous functions such that for all $x_{1}, x_{2}$ in $(a, b)$ and every $y_{1}, y_{2}$ there exists a unique member $h_{\alpha_{0} \beta_{0}}(x)$ of the family such that $h_{\alpha_{0} \beta_{0}}\left(x_{i}\right)=y_{i}(i=1,2)$. Then a function $g(x)$ is said to be a subfunction of the given family on $(a, b)$ provided $^{1}$ we have

$$
g\left[t x_{1}+(1-t) x_{2}\right] \leq h_{a_{1} \beta_{1}}\left[t x_{1}+(1-t) x_{2}\right]
$$

for all $x_{1}, x_{2}$ in $(a, b)$ and for all $t$ on the range $0 \leq t \leq 1$, and where

$$
h_{a_{1} \beta_{1}}\left(x_{i}\right)=g\left(x_{i}\right) \quad(i=1,2) .
$$

Geometrically, (1.12) indicates that in the subinterval $\left(x_{1}, x_{2}\right)$ no part of the graph of the curve $y=g(x)$ lies above the member of the parameter family joining the points $\left[x_{1}, g\left(x_{1}\right)\right]$ and $\left[x_{2}, g\left(x_{2}\right)\right]$. We note that if $g(x)$ is convex, it is a subfunction of the two-parameter family of nonvertical straight lines.

1.2. A fundamental theorem. Necessary and sufficient conditions that a function $g(x)$ be a subfunction of a certain type of two-parameter family have been obtained by Shniad [10]. The following lemma and theorem are due to him; proofs are included because of the fundamental use made of the theorem in subsequent developments.

LEMMA 1.1. If $\phi(x)$ is a positive continuous function of $x$, and $\psi(x)$ is a strictly increasing continuous function of $x$, on $a<x<b$, then the condition that $g(x)$ be a subfunction of the family $A \phi+B \phi \psi$, where $A$ and $B$ are parameters of the family, is equivalent to the condition that $g / \phi$ be a convex function of $\psi$.

Proof. The hypotheses on $\phi$ and $\psi$ ensure that $g / \phi$ is a continuous function of $\psi$. To prove the existence of a unique member of the family through any two points $\left(x_{i}, y_{i}\right)(i=1,2)$, with the $x_{i}$ distinct and in the interval, it suffices to note that

$$
\left|\begin{array}{ll}
\phi\left(x_{1}\right) & \phi\left(x_{1}\right) \psi\left(x_{1}\right) \\
\phi\left(x_{2}\right) & \phi\left(x_{2}\right) \psi\left(x_{2}\right)
\end{array}\right|=\phi\left(x_{1}\right) \phi\left(x_{2}\right)\left[\psi\left(x_{2}\right)-\psi\left(x_{1}\right)\right] \neq 0 .
$$

Let $x_{1}$ and $x_{2}$ satisfy $a<x_{1}<x_{2}<b$, and let

${ }^{1} \mathrm{~A}$ more general definition of the subfunction property is given in [5]; in [3] it is shown that a function satisfying (1.12) necessarily is continuous on $(a, b)$. 


$$
h_{\alpha_{1} \beta_{1}}(x)=A_{1} \phi(x)+B_{1} \phi(x) \psi(x)
$$

with

$$
h_{\alpha_{1} \beta_{1}}\left(x_{i}\right)=g\left(x_{i}\right)
$$

$$
(i=1,2) \text {. }
$$

Then the condition

$$
h_{a_{1} \beta_{1}}(x) \geq g(x) \quad \text { for } x_{1}<x<x_{2}
$$

is equivalent to the condition

$$
A_{1}+B_{1} \psi(x) \geq \frac{g(x)}{\phi(x)} \quad \text { for } x_{1}<x<x_{2}
$$

or that $g(x) / \phi(x)$ be a convex function of $\psi$ on the range $\psi(a+)<\psi<\psi(b-)$.

THEOREM 1.2. ${ }^{2}$ Let $\phi(x), \psi(x)$, and $g(x)$ be functions having the following properties on an interval $a<x<b$ :

a) the functions $\phi, \psi$, and g have continuous second derivatives,

b) the inequalities $\phi(x)>0$ and $\psi^{\prime}(x)>0$ hold, and

c) each of the functions $\phi(x)$ and $\phi(x) \psi(x)$ is a solution of the differential equation

$$
h^{\prime \prime}+P h^{\prime}+Q h=0 \text {, }
$$

where $P$ and $Q$ are continuous on the interval.

Then a necessary and sufficient condition that $g(x)$ be a subfunction of the family $A \phi+B \phi \psi$ on the given interval is that

$$
g^{\prime \prime}+P g{ }^{\prime}+Q g \geq 0
$$

on the interval.

Proof. From Lemma 1.1 it follows that $g$ is a subfunction of the family if and only if $g / \phi$ is a convex function of $\psi$. Since $g / \phi$ has a continuous second derivative with respect to $\psi$, the latter condition is equivalent to

2 The conclusion of this theorem is obtained in a more general setting in [9]. However, the proof is immediate for the theorem as stated here, and this form is sufficient for our purposes. 


$$
\frac{1}{\left(\psi^{\prime}\right)^{2} \phi}\left\{g^{\prime \prime}+g^{\prime}\left[-\frac{\psi^{\prime \prime}}{\psi^{\prime}}-\frac{2 \phi^{\prime}}{\phi}\right]+g\left[\frac{\psi^{\prime \prime} \phi^{\prime}}{\psi^{\prime} \phi}-\frac{\phi^{\prime \prime}}{\phi}+2\left(\frac{\phi^{\prime}}{\phi}\right)^{2}\right]\right\} \geq 0
$$

From the Wronskian relation we easily verify that $\phi$ and $\phi \psi$ are linearly independent solutions of the differential equation. Then the theorem follows from uniqueness properties of linearly independent solutions of this type of differential equation.

1.3. Sub- $K_{0}$ functions. The differential equation we are to consider is

$$
h^{\prime \prime}+K_{0} h=0 \text {, }
$$

where $K_{0}$ is a negative constant, and the interval of definition is $0 \leq x<b \leq \infty$. The two-parameter family of solutions of the equation is given by

$$
\left\{h_{\alpha \beta}(x)\right\}=\left\{\alpha \cosh \left(\sqrt{-K_{0}} x\right)+\beta \sinh \left(\sqrt{-K_{0}} x\right)\right\},
$$

where $\alpha$ and $\beta$ are the parameters. A property of this family is given in the following lemma; we omit the proof.

LeMmA 1.3. If A: $\left(x_{1}, y_{1}\right)$ and $\mathrm{B}:\left(x_{2}, y_{2}\right)$ are two points with $x_{1} \neq x_{2}$, then there is one and only one curve of the family $\left\{h_{\alpha \beta}(x)\right\}$ passing through $\mathrm{A}$ and B. Thus, if $y_{1} \geq 0$ and $y_{2} \geq 0$, the curve $h_{\alpha_{1} \beta_{1}}(x)$ passing through A and B satisfies $h_{a_{1} \beta_{1}}(x) \geq 0$ for $x_{1} \leq x \leq x_{2}$.

Definition. A function $g(x)$ will be said to be a sub-K $K_{0}$ function of $x$ if it is a subfunction of the family $\left\{h_{\alpha \beta}(x)\right\}$ of (1.31) on the interval $0 \leq x<$ $b \leq \infty$. Moreover, $g(x)$ will be said to be a $K_{0}$-function if the sign of equality of its subfunction relation (1.12) holds throughout the interval; and it will be a strictly sub- $K_{0}$ function if the strict inequality holds throughout for $0<t<1$.

It is convenient to introduce a second-order differential operator $\subseteq$ defined by

$$
\widetilde{\Xi} \equiv D^{2}+K_{0},
$$

where $K_{0}$ is a negative constant; we may write $\widetilde{\Xi}_{x}$ to indicate the variable for differentiation.

REMARK. With the choices

$$
\phi(x) \equiv \cosh \left(\sqrt{-K_{0}} x\right) \text { and } \psi(x) \equiv \tanh \left(\sqrt{-K_{0}} x\right),
$$


the family $\{\alpha \phi+\beta \phi \psi\}$ coincides with the family (1.31), and these functions $\phi$ and $\psi$ satisfy the hypotheses of Theorem 1.2. Hence a function $g(x)$ of class $C^{2}$ is a sub- $K_{0}$ function $\left(K_{0}\right.$-function) if and only if $\widetilde{\Xi}_{g}(x) \geq 0\left(\widetilde{\cup}_{g}(x)=0\right)$ on the interval.

Certain elementary properties of sub- $K_{0}$ functions are given in the following theorems. The proofs are omitted as they merely involve applying the foregoing remark to appropriate members of the family $\left\{h_{\alpha \beta}(x)\right\}$.

THEOREM 1.4. Any linear combination of sub- $K_{0}$ functions with nonnegative coefficients is a sub- $K_{0}$ function.

THEOREM 1.5. Let $f(x)$ be a nonnegative sub-K function, and let $k$ be a constant $\geq 1$. Then $[f(x)]^{k}$ is a sub-Ko function; in fact, $[f(x)]^{k}$ is a sub-kK function.

THEOREM 1.6. Let $f_{i}(x)(i=1,2, \cdots, n)$ be convex functions of $x$ which are nonnegative and monotonic nondecreasing and at least one of which is a sub- $K_{0}$ function. Then the product function $f_{1} f_{2} \cdots f_{n}$ is a sub-K $K_{0}$ function.

\section{Surfaces of Negative Curvature}

2.1. Geodesic parameters. Let an analytic surface $S$ be represented by geodesic parameters $[7$, p. 174] $(u, v)$, so that

$$
d s^{2}=d u^{2}+\mu^{2}(u, v) d v^{2}
$$

and

$$
d a=\mu(u, v) d u d v
$$

where the curves $v=$ constant are the geodesics, and the curves $u=$ constant are the geodesic parallels. The surface $S$ is said to be given in geodesic representation.

Singular points of the geodesic family are points where $\mu=0$; other points, where $\mu>0$, are regular points.

The Gaussian curvature $K$ of $S$ exists at all regular points. If $S$ is given in geodesic representation, the Gaussian curvature is given $[7, \mathrm{p} .181]$ by the formula

$$
K=-\frac{1}{\mu} \frac{\partial^{2} \mu}{\partial u^{2}}
$$


DEFINITION. An analytic surface $S$ will be said to be $a$ sub-Ko surface if its Gaussian curvature is bounded from above by $K_{0}$, a negative constant, at all regular points of $S$. Moreover, $S$ will be said to be $a K_{0}$-surface if its Gaussian curvature everywhere is $K_{0}$. If $S$ is a sub- $K_{0}$ surface which is not a $K_{0}$-surface, it will be said to be a strictly sub-K $K_{0}$ surface.

\subsection{Geodesic parallels. We have the following lemma.}

LEммA 2.1. If an analytic surface $S$ is given in geodesic representation, then a necessary and sufficient condition that $S$ be a sub- $K_{0}$ surface is that the function $\mu\left(u_{,} v_{0}\right)$ be a sub-K $K_{0}$ function of $u$ for each line-segment $u_{1}<u<u_{2}$, $v=v_{0}$ in the $(u, v)$-domain of definition.

Proof. The result follows directly from (2.13) and Lemma 1.3 by an argument analogous to that in [4, p.286]. The proof reveals that $\mu$ is a strictly sub- $K_{0}$ function of $u$ if and only if $S$ is a strictly sub- $K_{0}$ surface, and that $\mu$ is a $K_{0}$-function of $u$ if $S$ is a $K_{0}$-surface.

Let $S$ be a sub- $K_{0}$ surface given in geodesic representation. Then we have the following results.

THEOREM 2.2. Let the arcs $C(u)\left(u_{1} \leq u \leq u_{2}\right)$, of length $l(u)$, be arcs of geodesic parallels between geodesics $v=v_{1}$ and $v=v_{2}\left(v_{1}<v_{2}\right)$ on $S$. Then the length $l(u)$ is a sub- $K_{0}$ function of $u$ (that is, of the geodesic length

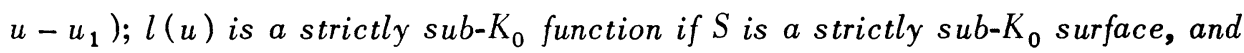
$l(u)$ is a $K_{0}$-function if $S$ is a $K_{0}$-surface.

Proof. A proof may be patterned on that of the related theorem in [4, p. 287], in which we substitute the appropriate member (which is of class $C^{2}$ ) of the family $(1.31)$ in a subfunction inequality in place of the convexity inequality.

THEOREM 2.3. Let the arcs $C(u)\left(u_{1}-\mathbb{W}<u<u_{1}+W\right)$, of length $l(u)$, be arcs of geodesic parallels between geodesics $v=v_{1}$ and $v=v_{2}\left(v_{1}<v_{2}\right)$ on $S$, and let $a(w)$ denote the area of the part of $S$ enclosed by $v=v_{1}, C\left(u_{1}+w\right)$, $v=v_{2}, C\left(u_{1}-w\right)(0 \leq w<W)$. Then $a(w)$ is a sub-Ko function of $w ; a(w)$ is a strictly sub- $K_{0}$ function if $S$ is a strictly sub-K $K_{0}$ surface, and is a $K_{0}$-function of $w$ if $S$ is a $K_{0}$-surface.

Proof. The proof is similar to that in [4, p.288] when we consider subfunction properties instead of convexity properties.

2.3. Geodesic polar coordinates. Let the analytic surface $S$ be represented 
in geodesic polar coordinates $[7, \mathrm{p} .181](u, v)$, that is, coordinates for which (2.11), (2.12), and

$$
\mu(0, v)=0,\left[\frac{\partial \mu}{\partial u}\right]_{u=0}=1
$$

are satisfied. The curve $u=u_{0}$ is a geodesic circle with center at the pole $P$ of the representation and geodesic radius $u_{0}$.

We shall write $r, \theta$ for $u, v$, respectively.

Hereafter we indicate functions determined by, or calculated for, a $K_{0}$ surface by a subscript zero. Some such functions can be determined explicitly.

Lemma 2.4. Let $S_{0}$ be a $K_{0}$-surface, and let $l_{0}(r)$ and $a_{0}(r)$ denote the circumference and area, respectively, of the geodesic circle on $S_{0}$ with fixed center $P$ and geodesic radius $r$. Then

$$
l_{0}(r) \equiv \frac{2 \pi}{\sqrt{-K_{0}}} \sinh \left(\sqrt{-K_{0}} r\right)
$$

and

$$
a_{0}(r) \equiv \frac{2 \pi}{-K_{0}}\left[\cosh \left(\sqrt{-K_{0}} r\right)-1\right]
$$

Moreover,

$$
l_{0}(r)>2 \pi r
$$

and

$$
a_{0}(r)>\pi r^{2}
$$$$
(r>0 \text { on } S) \text {. }
$$

Proof. Since $\widetilde{\Xi}_{u} \mu=0$, we find that the function $\mu_{0}(r)$ of the family (1.31) satisfying (2.31) is

$$
\mu_{0}(r)=\frac{1}{\sqrt{-K_{0}}} \sinh \left(\sqrt{-K_{0}} r\right)
$$

When we evaluate (2.11) and (2.12) for a geodesic circle using this expression for $\mu$, we obtain the formulas of the lemma. The inequalities are easily established; cf. [4, p. 291-292]. 
We remark that the functions $l_{0}(r)$ and $a_{0}(r)$ will occur in formulas which refer to a sub- $K_{0}$ surface $S$; in such cases, (2.32) and (2.33) provide definitions of these functions on $S$.

\section{Subfunctions for Geodesic Circles}

3.1. Definition. Some functions of geometrical significance involving the geodesic radius have certain properties in common. We collect these properties in the following definition.

Condition C. For a given sub- $K_{0}$ surface $S$ and for a given pole $P$ of geodesic polar coordinates on $S$, a function $\phi(r)$ of the geodesic radius $r$ satisfies Condition C provided: $\phi(0)=0$; for $r \geq 0$ on $S, \phi(r)$ is a continuous, nondecreasing sub- $K_{0}$ function of $r ; \phi(r) \equiv 0$ if $S$ is a $K_{0}$-surface, but otherwise $\phi(r)$ is a strictly sub- $K_{0}$ function.

If we let $K_{0}=0$, the $K_{0}$-surface becomes a developable surface, and the "sub- $K_{0}$ function of $r$ " property becomes the usual "convex function of $r$ " property. Thus our Condition C specializes to Condition A of [4, p. 289] when $K_{0}=0$.

It follows from the theorems of $\S \overline{1} .3$ that sums and products of functions which satisfy Condition C also satisfy Condition C.

3.2. The length function. Hereafter we assume that $\mu(r, \theta)$ is of class $C^{2}$, which ensures the existence of the derivatives we write. We now consider a geodesic circle $C_{r}$ on $S$ with fixed center $P$ and geodesic radius $r$.

Lemm A 3.1. Let $S$ be an analytic sub- $K_{0}$ surface, and let $l(r)$ denote the length of the circumference of $C_{r}$. Then $l(r)$ satisfies the differential relation

$$
S_{S} l(r) \equiv l^{\prime \prime \prime}(r)+K_{0} l(r) \geq 0 \quad(r \geq 0 \text { on } S) .
$$

Proof. The result is immediate since $\widetilde{G}_{r} \mu(r, \theta) \geq 0$ for $r \geq 0$ on $S$. We note that equality holds in (3.21) if and only if $S$ is a $K_{0}$-surface, that is, in our notation, if and only if we have $\subseteq l_{0}(r)=0$, where $l_{0}(r)$ is given by (2.32).

Lemma 3.2. Let $S$ be an analytic sub- $K_{0}$ surface, and let $a(r)$ denote the area of $C_{r}$. Then a $(r)$ satisfies the differential relation

$$
a^{\prime \prime}(r)+K_{0} a(r)-2 \pi \equiv l^{\prime}(r)+K_{0} a(r)-2 \pi \geq 0 \quad(r \geq 0 \text { on } S) .
$$

Proof. By differentiating the area function 


$$
a(r)=\int_{0}^{r} \int_{0}^{2 \pi} \mu(\rho, \theta) d \rho d \theta
$$

we get

$$
a^{\prime \prime}(r)=\int_{0}^{2 \pi} \frac{\partial \mu}{\partial r} d \theta \equiv l^{\prime}(r)
$$

Since $a(0)=0$, and $l^{\prime}(0)=2 \pi$ by $(2.31)$, we have equality in $(3.22)$ for $r=0$. The derivative of the function

$$
l^{\prime}(r)+K_{0} a(r)-2 \pi
$$

is $\widetilde{l} l(r)$, which is nonnegative by Lemma 3.1; hence the left member of (3.22) is monotonic nondecreasing, and (3.22) holds. It is readily seen that equality holds in (3.22) if and only if $S$ is a $K_{0}$-surface.

THEOREM 3.3. Let $S$ be an analytic sub-K $K_{0}$ surface, and let $l(r)$ denote the length of the circumference of $C_{r}$. Then the function

$$
\phi_{1}(r) \equiv l(r)-l_{0}(r) \quad(r \geq 0 \text { on } S),
$$

satisfies Condition C.

Proof. The functions $\mu(r, \theta)$ and $\mu_{0}(r, \theta)$ associated with the surfaces $S$ and $S_{0}$, respectively, both satisfy $(2.31)$, and are such that

$$
\begin{array}{ll}
\frac{\partial^{2} \mu}{\partial r^{2}}+K \mu=0 & (r \geq 0 \text { on } S), \\
\frac{\partial^{2} \mu_{0}}{\partial r^{2}}+K_{0} \mu_{0}=0 & (r \geq 0 \text { on } S),
\end{array}
$$

where $K \leq K_{0}$. By Sturm's oscillation theorems [8, Chap. X], it follows that

$$
\mu(r, \theta)-\mu_{0}(r, \theta) \geq 0 \quad(r \geq 0 \text { on } S),
$$

and

$$
\frac{\partial \mu}{\partial r}-\frac{\partial \mu_{0}}{\partial r} \geq 0 \quad(r \geq 0 \text { on } S)
$$


Hence we find that $\phi_{1}(0)=0, \phi_{1}(r) \equiv 0$ on $S$ if and only if $S$ is a $K_{0}$-surface, and

$$
\phi_{1}^{\prime}(r) \equiv l^{\prime}(r)-l_{0}^{\prime}(r)=\int_{0}^{2 \pi}\left(\frac{\partial \mu}{\partial r}-\frac{\partial \mu_{0}}{\partial r}\right) d \theta \geq 0 \quad(r \geq 0 \text { on } S) .
$$

Then calculation shows that

$$
\widetilde{\circlearrowleft} \phi_{1}(r)=\Xi l(r)
$$

whence

$$
\widetilde{S} \phi_{1}(r) \geq 0
$$

by Lemma 3.1. Thus, by Theorem 1.2, $\phi_{1}(r)$ satisfies Condition C.

COROLLARY 3.4. If $S$ is an analytic sub- $K_{0}$ surface, then $l(r)$ is a monotonic increasing sub- $K_{0}$ function of $r$ and satisfies the inequality

$$
l(r) \geq l_{0}(r) \quad(r \geq 0 \text { on } S)
$$

$l(r)$ is a strictly sub-K $K_{0}$ function if and only if $S$ is not a $K_{0}$-surface.

Proof. The inequality follows from Theorem 3.3 and the identity

$$
l(r)=l_{0}(r)+\phi_{1}(r) \quad(r \geq 0 \text { on } S) .
$$

REMARK. The function $\phi_{1}(r)$ may be modified to form a new function in the following way: replace the function $l_{0}(r)$ (Lemma 2.4) in $\phi_{1}(r)$ by its Maclaurin series expansion from which has been deleted any finite or infinite number of terms. The new function so obtained is a nonnegative, monotonic increasing, sub- $K_{0}$ function of $r$. In like manner, similar functions may be formed from subsequent $\phi$ functions which involve subtractive functions $l_{0}(r)$ and $a_{0}(r)$. We omit proofs.

3.3. The area function. $O n$ a surface where $K \leq K_{0}$, the area function $a(r)$ for a geodesic circle $C_{r}$ has properties similar to those given for $l(r)$.

THEOREM 3.5. Let $S$ be an analytic sub- $K_{0}$ surface, and let $a(r)$ denote the area of $C_{r}$. Then the function

$$
\phi_{2}(r) \equiv a(r)-a_{0}(r) \quad(r \geq 0 \text { on } S)
$$


satisfies Condition C.

Proof. Verification is immediate by use of Lemma 3.2 and Theorem 1.2.

COROLLARY 3.6. If $S$ is an analytic sub-K $K_{0}$ surface, then $a(r)$ is a monotonic increasing sub- $K_{0}$ function of $r$ and satisfies the inequality

$$
a(r) \geq a_{0}(r) \quad(r \geq 0 \text { on } S)
$$

$a(r)$ is a strictly sub-K $K_{0}$ function if and only if $S$ is not a $K_{0}$-surface.

Proof. The inequality follows from Theorem 3.5 and the identity

$$
a(r) \equiv a_{0}(r)+\phi_{2}(r) \quad(r \geq 0 \text { on } S) .
$$

We shall find additional theorems for the functions $a(r)$ and $\phi_{2}(r)$ showing certain subfunction properties of these functions when an additional assumption is made for the surface $S$. In the sequel we use the following lemma, which shows that certain conditions which clearly imply the sub- $K_{0}$ function property for a function also imply this property for its square root.

LEMмA 3.7. If $g(r)$ is a nonnegative function for which $g^{\prime \prime \prime}(r)$ exists in the interval $\alpha \leq r<\dot{\beta}$, and $g(r)$ satisfies

$$
h(\alpha) \equiv 2 g(\alpha) g^{\prime \prime}(\alpha)-\left[g^{\prime}(\alpha)\right]^{2}+4 K_{0}[g(\alpha)]^{2} \geq 0
$$

and

$$
g^{\prime \prime \prime}(r)+4 K_{0} g^{\prime}(r) \geq 0 \quad(\alpha \leq r<\beta),
$$

then $[g(r)]^{1 / 2}$ is a sub-Ko function in $\alpha \leq r<\beta$ and is a strictly sub- $K_{0}$ function there provided

$$
g^{\prime \prime}(r)+4 K_{0} g^{\prime}(r)>0 \quad(\alpha<r<\beta) .
$$

Proof. If we let $f(r) \equiv[g(r)]^{1 / 2}$, then at points where $f(r) \neq 0$ we have

$$
\widetilde{\circlearrowleft} f(r)=\frac{1}{4}[g(r)]^{-3 / 2} h(r) .
$$

Moreover,

$$
h^{\prime}(r)=2 g\left(g^{\prime \prime \prime}+4 K_{0} g^{\prime}\right),
$$


so that from the hypotheses we get

$$
h(\alpha) \geq 0, h^{\prime}(r) \geq 0
$$

whence $h(r) \geq 0$. Thus $\widetilde{S}(r) \geq 0$ at points where $f(r) \neq 0$. And, since the nonnegative function $f(r)$ satisfies the subfunction inequality (1.12) for points where $f(r)=0$, it follows that the continuous function $f(r)$ is a sub- $K_{0}$ function for $\alpha \leq r<\beta$.

With (3.31) and (3.32) the nonnegative sub- $K_{0}$ (and hence convex) function $g(r)$ can vanish at no more than one point of $\alpha \leq r<\beta$, whence, by (3.33), we have $h(r)>0(\alpha<r<\beta)$. It follows that we have $\mathscr{S} f(r)>0$ except for at most one point of $\alpha<r<\beta$, so that $f(r)$ is a strictly sub- $K_{0}$ function for $\alpha \leq r<\beta$. This completes the proof of the lemma.

An additional assumption on the surface $S$ causes certain functions immediately to satisfy (3.33) for $r \geq 0$ on $S$. Thus, if $S$ satisfies $K \leq 4 K_{0}$ for its Gaussian curvature, then a modification of the proof of Theorem 3.3 indicates that we have

$$
a^{\prime \prime \prime}(r)+4 K_{0} a^{\prime}(r) \equiv l^{\prime \prime}(r)+4 K_{0} l(r) \geq 0 \quad(r \geq 0 \text { on } S),
$$

with equality holding if and only if $S$ is a $4 K_{0}$-surface. We now determine some functions which have certain subfunction properties in common; these properties are collected in:

Condition D. For a given sub- $4 K_{0}$ surface $S$ and for a given pole $P$ of the geodesic polar coordinates on $S$, a function $\psi(r)$ of the geodesic radius $r$ satisfies Condition D provided: $\psi(0)=0$; for $r \geq 0$ on $S, \psi(r)$ is a continuous monotonic nondecreasing sub- $K_{0}$ function of $r$; and $\psi(r)$ is a strictly sub- $K_{0}$ function except possibly when $S$ is a $4 K_{0}$-surface.

THEOREM 3.8. Let $S$ be an analytic sub-4 $K_{0}$ surface, and let $a(r)$ denote the area of the geodesic circle $C_{r}$. Then

$$
\psi_{1}(r) \equiv[a(r)]^{1 / 2}
$$

and

$$
\psi_{2}(r) \equiv\left[\phi_{2}(r)\right]^{1 / 2} \equiv\left[a(r)-a_{0}(r)\right]^{1 / 2}
$$

satisfy Condition $\mathrm{D}$, and $\psi_{1}(r)$ is a $K_{0^{-}}$function if $S$ is a $4 K_{0^{-}}$-surface. 
Proof. We have

$$
a^{\prime}(r)=l(r), a^{\prime \prime}(r)=l^{\prime}(r), a^{\prime \prime \prime}(r)=l^{\prime \prime}(r) ;
$$

hence, beside $a(0)=0$, we have

$$
a^{\prime}(0)=0, a^{\prime \prime \prime}(r) \geq 0 \quad(r \geq 0 \text { on } S),
$$

with

$$
a^{\prime \prime \prime}(r)+4 K_{0} a^{\prime}(r)>0
$$

for $r>0$ on $S$ unless $K \equiv 4 K_{0}$. Then for $r \geq 0$ on $S, a(r)$ satisfies the hypotheses on $g(r)$ of Lemma 3.7, so that $\psi_{1}(r)$ satisfies Condition D for $r \geq 0$ on $S$, and is a strictly sub- $K_{0}$ function if $S$ is not a $4 K_{0}$-surface. If $S$ is a $4 K_{0^{-}}$surface, then

$$
[a(r)]^{1 / 2} \equiv\left[\frac{2 \pi}{-4 K_{0}}\left\{\cosh \left(\sqrt{-4 K_{0}} r\right)-1\right\}\right]^{1 / 2} \equiv\left(\frac{\pi}{-K_{0}}\right)^{1 / 2} \sinh \left(\sqrt{-K_{0}} r\right)
$$

and thus it is a $K_{0}$-function.

The proof for $\psi_{2}(r)$ is similar in method and is omitted.

We can find other functions which satisfy Condition D. Let $l_{1}(r)$ and $a_{1}(r)$ denote the length of circumference and area, respectively, of the geodesic circle $C_{r}$ on a $4 K_{0}$-surface. Formulas for $l_{1}(r)$ and $a_{1}(r)$ can be written (see Lemma 2.4), and these expressions serve to define $l_{1}(r)$ and $a_{1}(r)$ for a surface $S$ having arbitrary curvature. If $S$ is a sub- $4 K_{0}$ surface, then, by methods analogous to those of Lemma 3.1 and Lemma 3.2, we find the relations

$$
l^{\prime}(r) \geq 2 \pi-4 K_{0} a(r) \quad(r \geq 0 \text { on } S),
$$

and

$$
l^{\prime \prime}(r)+4 K_{0} l(r) \geq 0 \quad(r \geq 0 \text { on } S),
$$

with the equality sign holding for $r>0$ on $S$ if and only if $S$ is a $4 K_{0}$-surface; that is,

$$
l_{1}^{\prime}(r)=2 \pi-4 K_{0} a_{1}(r)
$$

and 


$$
l_{1}^{\prime \prime}(r)+4 K_{0} l_{1}(r)=0
$$

THEOREM 3.9. Let $S$ be an analytic sub- $4 K_{0}$ surface, and let $l(r)$ and $a(r)$ denote the circumference and area function, respectively, of $C_{r}$ on $S$. Then the functions

$$
\begin{aligned}
& \psi_{3}(r) \equiv l(r)-l_{1}(r), \\
& \psi_{4}(r) \equiv a(r)-a_{1}(r),
\end{aligned}
$$

and

$$
\psi_{5}(r) \equiv\left[a(r)-a_{0}(r)\right]^{1 / 2}
$$

satisfy Condition D.

Proof. The method is that used in earlier theorems wherein now we apply the four relations which immediately precede Theorem 3.9.

REMARK. It was indicated earlier that our Condition $\mathrm{C}$ reduces to Condition A of [4, p. 289] if $K_{0}=0$. Now if $K_{0}=0$, the assumption that $S$ satisfies $K \leq 4 K_{0}$ imposes no new requirement upon the surface. In fact, our Condition D becomes Condition A if $K_{0}=0$ and if the function $\psi(r)$ is identically zero when the surface is developable.

The role played by the condition $K \leq 4 K_{0}$, when $K_{0} \neq 0$ for "square root" functions is indicated in the following theorem.

THEOR EM 3.10. Let $S$ be an analytic sub- $K_{0}$ surface, and let $a(r)$ denote the area of $C_{r}$ on $S$. Then in order that the function

$$
\psi_{1}(r) \equiv[a(r)]^{1 / 2}
$$

be a sub- $K_{0}$ function of $r$ for every possible pole $P$, it is necessary and sufficient that $S$ be a sub- $4 K_{0}$ surface.

Proof. The sufficiency has been established in Theorem 3.8.

Now let $P_{1}$ be a point of $S$ where $K_{1}>4 K_{0}$, and let $P_{1}$ be the pole of a geodesic polar coordinate system. Since $S$ is analytic, there exists a neighborhood of $P_{1}$ in which $K>4 K_{0}$, and hence a value $r_{1}>0$ such that the geodesic circle of radius $r_{1}$ lies entirely within this neighborhood. In this coordinate system we have 


$$
\frac{\partial^{2} \mu}{\partial r^{2}}+4 K_{0} \mu<0
$$

and then it easily follows that

$$
l^{\prime \prime}(r)+4 K_{0} l(r)<0 \quad\left(0<r \leq r_{1} \text { on } S\right) .
$$

By calculation we get that

$$
\widetilde{S} \psi_{1}(r)=\frac{1}{4} a^{-3 / 2}\left[2 a l^{\prime}-l^{2}+4 K_{0} a^{2}\right] \equiv \frac{1}{4} a^{-3 / 2} h(r),
$$

where $h(r)$ is the bracketed expression. Then we have that $h(0)=0$, and

$$
h^{\prime}(r)=2 a\left(l^{\prime \prime}+4 K_{0} l\right)<0
$$$$
\left(0<r \leq r_{1} \text { on } S\right)
$$

hence $h(r)<0$ for $0<r \leq r_{1}$, and thus also

$$
\Im \psi_{1}(r)<0
$$

$$
\left(0<r \leq r_{1} \text { on } S\right)
$$

Then by Theorem 1.2, $\psi_{1}(r)$, when evaluated in a coordinate system with such a pole, cannot be a sub- $K_{0}$ function.

\section{The Isoperimetric Inequality and Related Functions}

4.1. The isoperimetric inequality. Let $L$ and $A$ denote the perimeter and area, respectively, of a simply connected region bounded by an analytic curve on a surface of nonpositive curvature. The isoperimetric inequality

$$
\theta \equiv \frac{L^{2}}{4 \pi}-A \geq 0
$$

holds for such a region. In fact, the following theorem [6, p. 670-672] has been established:

For an analytic surface $S$, a necessary and sufficient condition that (4.11) hold for all simply connected regions bounded by analytic curves on $S$ is that $K \leq 0$ on $S$. Further, if $K \leq 0$ but $K \not \equiv 0$ on $S$, then the strict sign of inequality holds in (4.11); while if $K \equiv 0$ on $S$, then the sign of equality holds in (4.11) only for geodesic circles on $S$. 
We shall study the function $\theta$ of (4.11) and some modifications of it for sub- $K_{0}$ function properties when $S$ is assumed to be a sub- $K_{0}$ surface and the region is that determined by a geodesic circle. A well-known generalization of the function $\theta$ for geodesic circles on surfaces of constant negative curvature $K_{0}$ is the function

$$
\phi_{3}(r) \equiv \frac{l^{2}(r)}{4 \pi}+\frac{K_{0} a^{2}(r)}{4 \pi}-a(r)
$$

which we shall call the isoperimetric function.

THEOREM 4.1. Let $S$ be an analytic sub- $K_{0}$ surface, and let $l(r)$ denote the length of the circumference, and $a(r)$ the area, of the geodesic circle $C_{r}$ on $S$. Then the isoperimetric function $\phi_{3}(r)$ satisfies Condition $\mathrm{C}$.

Proof. Squaring the inequality (3.22) and using (3.21), we obtain

$$
4 \pi \Im \phi_{3}(r) \geq\left[l^{\circ 2}+K_{0} l^{2}-4 \pi^{2}\right] \equiv h(r),
$$

where $h(r)$ is the function in brackets. Then we see that $h(0)=0$, and that $h^{\prime}(r) \geq 0$ for $r \geq 0$ by (3.21); hence $\Im_{\phi_{3}}(r) \geq 0$ for $r \geq 0$ on $S$, and thus $\phi_{3}(r)$ is a sub- $K_{0}$ function by Theorem 1.2. The other requirements of Condition $\mathrm{C}$ are easily found to be satisfied by $\phi_{3}(r)$.

COROLLARY 4.2. If $S$ is an analytic sub- $K_{0}$ surface, and $l(r)$ and $a(r)$ as in the theorem, then the function

$$
\theta_{1}(r) \equiv \frac{l^{2}(r)}{4 \pi}-a(r)
$$

is a continuous monotonic nondecreasing sub- $K_{0}$ function of $r$.

Proof. It follows from the proof of Lemma 3.2 that $a(r)$ is a continuous monotonic nondecreasing sub- $K_{0}$ function of $r$, and then that $a^{2}(r)$ also has these properties by Theorem 1.5. Then, using the positive coefficient $-K_{0} / 4 \pi$, we may apply Theorem 1.4 and get

$$
\theta_{1}(r) \equiv \phi_{3}(r)-\frac{K_{0} a^{2}(r)}{4 \pi}
$$

so that $\theta_{1}(r)$ has the properties stated in Corollary 4.2 . 
COROLLARY 4.3. If $S$ is an analytic sub- $K_{0}$ surface, then

$$
\frac{l^{2}(r)}{4 \pi}-a(r)>\frac{l^{2}(r)}{4 \pi}+\frac{K_{0} a^{2}(r)}{4 \pi}-a(r) \geq 0 \quad(r>0 \text { on } S),
$$

where the sign of equality holds for $r>0$ on $S$ if and only if $S$ is a $K_{0}$-surface.

Proof. This corollary is an immediate consequence of Theorem 4.1 and Corollary 4.2 .

4.2. Modifications of the isoperimetric function. We shall consider modifications of the isoperimetric function, $\phi_{3}(r)$, which are produced by adding certain functions to it and/or by replacing $l(r)$ by $l_{0}(r)$ or $2 \pi r$ and $a(r)$ by $a_{0}(r)$ or zero. For example, the function $\theta_{1}(r)$ may be considered a modification of $\phi_{3}(r)$ formed by replacing the $a^{2}(r)$ function in $\phi_{3}(r)$ by zero.

THEOREM 4.4. Let $S$ be an analytic sub- $K_{0}$ surface, and let $l(r), l_{0}(r)$, and $a(r), a_{0}(r)$ denote length and area functions associated with the geodesic circle $C_{r}$. Then the functions

$$
\phi_{4}(r) \equiv \frac{l_{0}(r) l(r)}{4 \pi}+\frac{K_{0} a_{0}^{2}(r)}{4 \pi}-a(r)
$$

and

$$
\phi_{5}(r) \equiv \frac{l_{0}(r) l(r)}{4 \pi}+\frac{K_{0} a_{0}(r) a(r)}{4 \pi}-\frac{a_{0}(r)}{2}-\frac{a(r)}{2}
$$

satisfy Condition C.

Proof. We establish the result that

$$
l_{0} l^{\prime}-l l_{0}^{\prime} \equiv l_{0} l^{\prime}+K_{0} a_{0} l-2 \pi l \geq 0 \quad(r \geq 0 \text { on } S) .
$$

The function on the left is zero when $r=0$, and its derivative is the nonnegative (by Lemma 3.1) function $l_{0}(r) \Im l(r)$; hence (4.21) holds.

Now $\phi_{4}(0)=0$, and $\phi_{4}^{\prime}(r) \geq 0$ by $(4.21)$ and Theorem 3.3; thus $\phi_{4}(r)$ is monotonic nondecreasing. The calculation for $\widetilde{S} \phi_{4}(r)$ may be arranged so that

$$
\begin{aligned}
4 \pi \widetilde{S} \phi_{4}(r) \equiv\left[l_{0} \Xi l-K_{0} l\left(l-l_{0}\right)-2 K_{0} a_{0}\left(l^{\prime}-l_{0}^{\prime}\right)\right. \\
\left.-4 \pi K_{0}\left(a-a_{0}\right)+K_{0}\left(l_{0}^{2}+K_{0} a_{0}^{2}-4 \pi a_{0}\right)\right] .
\end{aligned}
$$


Then we have $\widetilde{S} \phi_{4}(r) \geq 0$ for $r \geq 0$ on $S$, since each parenthesis above is nonnegative by previous results - the last one, in particular, being identically zero according to Corollary 4.3. Thus $\phi_{4}(r)$ is a sub- $K_{0}$ function by Theorem 1.2. Finally, $\phi_{4}(r)$ satisfies Condition $\mathrm{C}$ since the signs of equality hold in the relations above if and only if $S$ is a $K_{0}$-surface, and obviously $\phi_{4}(r) \equiv 0$ if $S$ is a $K_{0}$-surface.

For $\phi_{5}(r)$, we find that $\phi_{5}(0)=0$ and that $\phi_{5}^{\prime}(r) \geq 0$ by Lemma 3.2. We may arrange the calculation so that

$$
\begin{aligned}
4 \pi \circlearrowleft \phi_{5}(r) & \equiv\left[l_{0} \Xi l+\left(l_{0}^{\prime} l^{\prime}+K_{0} l_{0} l-2 \pi l_{0}^{\prime}-2 \pi K_{0} a_{0}\right)\right] \\
& \geq l_{0}^{\prime} l^{\prime}+K_{0} l_{0} l-4 \pi^{2} \equiv h(r),
\end{aligned}
$$

where $h(r)$ is the function after the inequality sign. Clearly $h(0)=0$, and we find that

$$
h^{\prime}(r) \equiv l_{0}^{\prime} ธ l \geq 0
$$

Hence, $h(r) \geq 0$ for $r \geq 0$ on $S$, and then Theorem 1.2 ensures that $\phi_{5}(r)$ is a sub- $K_{0}$ function. The other conditions to complete the proof are easily verified.

Theorem 4.4 then admits a corollary which is analogous to the isoperimetric inequality for the functions $\phi_{4}(r)$ and $\phi_{5}(r)$; we omit its statement, but we remark that the inequality for $\phi_{5}(r)$ is sharper than the isoperimetric inequality (4.13) in that it presents a better estimate (greater lower bound) for $l(r)$.

The next theorem presents another function determined by the modification process.

THEOREM 4.5. For a surface and functions as in Theorem 4.4, the function

$$
\phi_{6}(r) \equiv \frac{r l(r)}{2}-a(r)-\frac{r l_{0}(r)}{2}+a_{0}(r) \equiv \frac{r}{2}\left[l(r)-l_{0}(r)\right]-\left[a(r)-a_{0}(r)\right]
$$

satisfies Condition C.

We omit the computations and also the corollary stating the inequality satisfied by $\phi_{6}(r)$.

It may be noted that with $\phi_{6}(r)$ satisfying Condition $\mathrm{C}$ it readily follows that $\phi_{4}(\dot{r})$ does. For if the function 


$$
\frac{1}{4 \pi}\left(l_{0}-2 \pi r\right)\left(l-l_{0}\right)
$$

which satisfies Condition C (in part by Theorem 1.6), is added to $\phi_{6}(r)$, we obtain $\phi_{4}(r)$, which then satisfies Condition C (in part by Theorem 1.4).

Theorem 4.5 suggests a consideration of the substitution of $l(r)-l_{0}(r)$ and $a(r)-a_{0}(r)$ for the functions $l(r)$ and $a(r)$. When this substitution is made in the isoperimetric function, we find that the new function does not satisfy our conditions. Nevertheless, in the next theorem we have a result of this procedure.

THEOREM 4.6. For a surface and functions as in Theorem 4.4, the function

$$
\phi_{7}(r) \equiv \frac{1}{4 \pi}\left[l(r)-l_{0}(r)\right]^{2}+\frac{K_{0}}{4 \pi}\left[a(r)-a_{0}(r)\right]^{2}
$$

satisfies Condition C.

Proof. We find that $\phi_{7}(0)=0$ and that $\phi_{7}^{\prime}(r) \geq 0$ by Lemma 3.2 and Theorem 3.3; thus $\phi_{7}(r)$ is monotonic nondecreasing. By computation we find that

$$
4 \pi \Im \phi_{7}(r) \geq\left[2\left(l^{\prime}-l_{0}^{\prime}\right)\left(l^{\prime}+K_{0} a-2 \pi\right)+K_{0}\left(l-l_{0}\right)^{2}+K_{0}^{2}\left(a-a_{0}\right)^{2}\right] \equiv h(r),
$$

where $h(r)$ is the bracketed expression. We see that $h(0)=0$, and that its derivative satisfies

$$
\begin{aligned}
h^{\prime}(r) & \geq 2\left[\left(l^{\prime \prime}-l_{0}^{\prime \prime}\right)\left(l^{\prime}+K_{0} a-2 \pi\right)+K_{0}\left(l-l_{0}\right)\left(l^{\prime}-l_{0}^{\prime}\right)+K_{0}^{2}\left(a-a_{0}\right)\left(l-l_{0}\right)\right] \\
& =2\left(l^{\prime \prime}+K_{0} l\right)\left(l^{\prime}+K_{0} a-2 \pi\right) \geq 0
\end{aligned}
$$

by Lemmas 3.1 and 3.2. Hence $h(r) \geq 0$ for $r \geq 0$ on $S$, with equality holding if and only if $S$ is a $K_{0}$-surface. It follows that $\phi_{7}(r)$ satisfies Condition C since obviously $\phi_{7}(r) \equiv 0$ if $S$ is a $K_{0}$-surface.

Theorem 4.6 admits refinements of the inequalities which appear in Corollary 4.3 and Corollary 3.4.

COROLLARY 4.7. Let an analytic sub- $K_{0}$ surface $S$ be referred to a geodesic polar coordinate system with pole $P$. Then, for geodesic circles, the isoperimetric function $\phi_{3}(r)$ and the functions $\phi_{5}(r)$ and $\phi_{7}(r)$ satisfy the inequalities 


$$
\phi_{3}(r) \geq 2 \phi_{5}(r) \geq 0
$$

and

$$
\phi_{3}(r) \geq \phi_{7}(r) \geq 0
$$

where the signs of equality hold for $r>0$ on $S$ if and only if $S$ is a $K_{0}$-surface, in which case all functions are identically zero.

Proof. It is easily seen that

$$
\phi_{7}(r) \equiv \phi_{3}(r)-2 \phi_{5}(r)
$$

and the corollary then follows from Theorems 4.6 and 4.3.

CoROLl ARY 4.8. Let an analytic sub- $K_{0}$ surface $S$ be referred to a geodesic polar coordinate system with pole $P$. Then the length of the circumference of a geodesic circle of radius $r$ satisfies the inequality

$$
l(r) \geq l_{0}(r)+\sqrt{-K_{0}}\left[a(r)-a_{0}(r)\right],
$$

where the sign of equality holds for $r>0$ on $S$ if and only if $S$ is a $K_{0}$-surface.

Proof. Since $K_{0}<0,4 \pi \phi_{7}(r)$ has real factors. The factor $\phi_{8}(r)$, where $\phi_{8}(r) \equiv l-l_{0}+\sqrt{-K_{0}}\left(a-a_{0}\right)$, satisfies $\phi_{8}(r) \geq 0$ by Corollaries 3.4 and 3.6; hence so also does the other factor by Corollary 4.7. This other factor yields (4.23).

Less precise relations may be obtained from the isoperimetric function by using the theorems of $\S 1.3$.

THEOREM 4.9. Let $S$ be an analytic sub- $K_{0}$ surface with length and area functions relating to geodesic circles on $S$ as previously defined. Then the functions

$$
\begin{gathered}
\phi_{9}(r) \equiv \frac{1}{4 \pi}\left[l^{2}(r)-l_{0}^{2}(r)\right]-\left[a(r)-a_{0}(r)\right], \\
\phi_{10}(r) \equiv \frac{1}{4 \pi}\left[l^{2}(r)-l_{0}^{2}(r)\right]+\frac{K_{0}}{4 \pi}\left[a^{2}(r)-a_{0}^{2}(r)\right],
\end{gathered}
$$

and 


$$
\phi_{11}(r) \equiv l^{2}(r)-\frac{a(r)}{a_{0}(r)} l_{0}^{2}(r) \equiv l^{2}(r)-4 \pi a(r) \cosh \left(\frac{\sqrt{-K_{0}} r}{2}\right)
$$

satisfy Condition C.

Proof. We refer to $\oint 1.3$ and merely indicate the verification of the desired subfunction property of these functions. Thus, $\phi_{g}(r)$ results from adding the function $-\left(K_{0} / 4 \pi\right)\left[a^{2}(r)-a_{0}^{2}(r)\right]$, which satisfies Condition $\mathrm{C}$, to the isoperimetric function $\phi_{3}(r)$. The function $\phi_{10}(r)$ is obtained by adding the function $\phi_{2}(r) \equiv a(r)-a_{0}(r)$ to $\phi_{3}(r)$. And the function $\phi_{11}(r)$ is obtained by adding $-K_{0} a(r)\left[a(r)-a_{0}(r)\right]$, which satisfies Condition C, to $4 \pi \phi_{3}(r)$.

4.3. Another kind of modification. The properties of the isoperimetric function and its modifications which we have developed now enable us to introduce new functions which satisfy our conditions. These new functions are produced by replacing each term of an expression by its square root.

THEOREM 4.10. Let $S$ be an analytic sub- $4 K_{0}$ surface with length and area functions relating to a geodesic circle on $S$ as previously defined. Then the functions

$$
\psi_{6}(r) \equiv \sqrt{a(r)}-\sqrt{a_{0}(r)}
$$

and

$$
\psi_{7}(r) \equiv \sqrt{a(r)}-\sqrt{a_{1}(r)}
$$

satisfy Condition D.

Proof. We have $\psi_{7}(0)=0$, and

$$
\psi_{7}^{\prime}(r)=\frac{1}{2}\left[\frac{l}{\sqrt{a}}-\frac{l_{1}}{\sqrt{a_{1}}}\right] \geq 0
$$

for $r \geq 0$ on $S$ by the properties of $\phi_{11}(r)$ of Theorem 4.9, since now $l_{1}(r)$ and $a_{1}(r)$ behave analogously to $l_{0}(r)$ and $a_{0}(r)$ of that theorem. Hence $\psi_{7}(r)$ is a monotonic nondecreasing function of $r$. Then using (3.34) and the isoperimetric identity satisfied by $l_{1}(r)$, we get

$$
\Xi \psi_{7}(r)=\frac{a^{-3 / 2}}{4}\left[2 a l^{\prime}-l^{2}+4 K_{0} a^{2}\right] \geq 0
$$


for $r \geq 0$ on $S$, since the function in brackets is identical with that which would occur for the function $\psi_{1}(r)$ of Theorem 3.8. Thus $\psi_{7}(r)$ satisfies Condition D.

The proof for $\psi_{6}(r)$ is similar to this for $\psi_{7}(r)$.

The next theorem presents a modification of the function $\phi_{11}(r)$ of Theorem 4.9.

THEOREM 4.11. Let $S$ be an analytic sub- $K_{0}$ surface with length and area functions relating to geodesic circles on $S$ as previously defined. Then the function

$$
\phi_{12}(r) \equiv l(r) \sqrt{a_{0}(r)}-l_{0}(r) \sqrt{a(r)}
$$

satisfies Condition C.

Proof. We first establish the inequality

$$
2 a(r) l^{\prime}(r)-l^{2}(r)+K_{0} a^{2}(r) \geq 0 \quad(r \geq 0 \text { on } S),
$$

where the sign of equality holds for $r>0$ on $S$ if and only if $S$ is a $K_{0}$-surface. The result is immediate, since the function on the left in (4.31) is zero at $r=0$, and its derivative is nonnegative for $r \geq 0$.

Clearly $\phi_{12}(0)=0$, and $\phi_{12}(r) \geq 0$ for $r>0$ on $S$ since $\phi_{11}(r)$ satisfies Condition C. Then, by substituting for $l^{\prime}(r)$ and $l_{0}^{\prime}(r)$ from (4.31), we find that

$$
\phi_{12}^{\prime}(r) \geq \frac{1}{2}\left[\left(\frac{l}{a}+\frac{l_{0}}{a_{0}}\right)\left(l \sqrt{a_{0}}-l_{0} \sqrt{a}\right)-K_{0} \sqrt{a a_{0}}\left(\sqrt{a}-\sqrt{a_{0}}\right)\right] \geq 0 .
$$

Inus $\phi_{12}(r)$ is monotonic nondecreasing. Then using (3.21) we find that

$$
\begin{aligned}
25 \phi_{12}(r) \geq\left(\frac{l^{\prime} l_{0}}{\sqrt{a_{0}}}-\frac{l l_{0}^{\prime}}{\sqrt{a}}\right) & +\left(\frac{l_{0}^{\prime} l}{\sqrt{a_{0}}}-\frac{l_{0}^{2} l}{2 a_{0} \sqrt{a_{0}}}-\frac{l_{0}^{\prime} l}{\sqrt{a}}\right) \\
& +\left(\frac{l^{\prime} l_{0}}{\sqrt{a_{0}}}-\frac{l^{\prime} l_{0}}{\sqrt{a}}+\frac{l^{2} l_{0}}{2 a \sqrt{a}}\right) .
\end{aligned}
$$

Now using $(4.31)$ in the last two parentheses, we get 


$$
\begin{aligned}
4 \circlearrowleft \phi_{12}(r) & \geq 2\left(\frac{l^{\prime} l_{0}}{\sqrt{a_{0}}}-\frac{l l_{0}^{\prime}}{\sqrt{a}}\right)+\frac{l l_{0}}{a a_{0}}\left(l \sqrt{a_{0}}-l_{0} \sqrt{a}\right)-K_{0} l_{0} \sqrt{\frac{a}{a_{0}}}\left(\sqrt{a}-\sqrt{a_{0}}\right) \\
& \geq \frac{2}{\sqrt{a}}\left(l^{\prime} l_{0} \sqrt{\frac{a}{a_{0}}}-l l_{0}^{\prime}\right) \geq \frac{2}{\sqrt{a}}\left(l^{\prime} l_{0}-l l_{0}^{\prime}\right) .
\end{aligned}
$$

Hence, by (4.21), it follows that $\widetilde{S}_{\phi_{12}}(r) \geq 0$ for $r \geq 0$ on $S$. Thus, on citing Theorem 1.2 and the obvious fact that $\phi_{12}(r) \equiv 0$ if $S$ is a $K_{0}$-surface, we have shown that $\phi_{12}(r)$ satisfies Condition C.

\section{Extensions AND GENERALIZATIONS}

5.1. Geodesic circular sectors. The generalization from a basic configuration of geodesic circles to one of geodesic circular sectors is indicated in [4, p. 296], and its relations apply immediately to this study.

We state some representative results.

THEOREM 5.1. Let $S$ be an analytic sub-K $K_{0}$ surface, and let $l\left(r ; \theta_{1}, \theta_{2}\right)$ and $a\left(r ; \theta_{1}, \theta_{2}\right)$ denote respectively the length of the bounding arc and the area of the geodesic circular sector on $S$ with fixed pole $P$, fixed angle from $\theta_{1}$ to $\theta_{2}, \theta_{1}<\theta_{2}$, and geodesic radius $r$. Then the functions

$$
\begin{aligned}
& \phi_{13}(r) \equiv l\left(r ; \theta_{1}, \theta_{2}\right)-l_{0}\left(r ; \theta_{1}, \theta_{2}\right), \\
& \phi_{14}(r) \equiv a\left(r ; \theta_{1}, \theta_{2}\right)-a_{0}\left(r ; \theta_{1}, \theta_{2}\right),
\end{aligned}
$$

and

$$
\begin{array}{r}
\phi_{15}(r) \equiv \frac{l^{2}\left(r ; \theta_{1}, \theta_{2}\right)}{2\left(\theta_{2}-\theta_{1}\right)}+\frac{K_{0} a^{2}\left(r ; \theta_{1}, \theta_{2}\right)}{2\left(\theta_{2}-\theta_{1}\right)}-a\left(r ; \theta_{1}, \theta_{2}\right), \\
\quad\left(r \geq 0, \theta_{1} \leq \theta \leq \theta_{2} \text { on } S\right),
\end{array}
$$

satisfy Condition $\mathrm{C}$.

The proof for each function is similar to the proof of the analogous result for the corresponding function for geodesic circles, and will not be given here.

Other functions which satisfy Condition C or Condition D for geodesic circular sectors (the analogues of those for geodesic circles) obviously could be 
written. It is clear that, as corollaries, we then obtain certain inequality relations between the length and area functions for a suitably restricted surface.

5.2. Regular super- $K_{0}$ surfaces. The preceding results concerning sub- $K_{0}$ surfaces hold in the large and are unaffected by singular points. We now describe somewhat analogous results for surfaces whose Gaussian curvature satisfies $K \geq K_{0}$; such surfaces will be called super- $K_{0}$ surfaces. We still assume $K_{0}<0$, although some of the results hold, in the small, for $K_{0}$ any constant. In general, our results will hold only on parts of $S$ where there are no singular points of the surface, or of the family of geodesics, other than at the pole of geodesic polar coordinates; and some of the results hold only in the small even where there are no singular points.

A function $f(x)$ is said to be a super- $K_{0}$ function provided $-f(x)$ is a sub- $K_{0}$ function.

A surface $S$ given in geodesic coordinates, or in geodesic polar coordinates, will be said to be regular provided there are no singular points on $S$ except, in the case of geodesic polar coordinates, at the pole $P$.

Lemma 2.1 holds if we add the restriction that $S$ is regular, and replace "sub- $K_{0}$ " by "super- $K_{0}$." Theorems 2.2 and 2.3 hold with the same alterations, and the inequality relations given by (3.21), (3.22), and (4.21) hold with the inequality signs'reversed.

THEOREM 5.2. Let $S$ be a regular analytic super- $K_{0}$ surface, and let $l(r)$ and $a(r)$ denote the length and area functions for a geodesic circle $C_{r}$. Then the functions $-\phi_{j}(r)(j=1,2,4,5,6,8)$ satisfy Condition C.

Proof. The theorem follows in routine fashion by an examination of earlier calculations for these functions in relation to (3.21), (3.22), and (4.21) with the inequality signs reversed.

Now consider the isoperimetric function $\phi_{3}(r)$. We compute $\phi_{3}^{\prime}(r)$, and find that $\phi_{3}(r)$ is monotonic nonincreasing on any regular super- $K_{0}$ surface $S$, and is monotonic decreasing if $S$ is not a $K_{0}$-surface. Actually, since $l^{\prime}(0)=2 \pi$, it follows from a consideration of $\widetilde{S}_{\phi_{3}}(r)$ that there is an $r_{0}=r_{0}(S, P)$ such that $\phi_{3}(r)$ is a super- $K_{0}$ function for $0 \leq r \leq r_{0}$.

From the properties of the functions $\phi_{j}(r)$ we obtain results for $l(r)$ and $a(r)$. We have

$$
l(r)=l_{0}(r)+\phi_{1}(r),
$$




$$
l^{\prime}(r)=l_{0}^{\prime}(r)+\phi_{1}^{\prime}(r)
$$

and

$$
\widetilde{\circlearrowleft} l(r)=\widetilde{\circlearrowleft} \phi_{1}(r) .
$$

Since the functions $-\phi_{j}(r)$ satisfy Condition $\mathrm{C}$ on regular super- $K_{0}$ surfaces, we have

$$
\phi_{1}(r) \leq 0, \tilde{\circlearrowleft} \phi_{1}(r) \leq 0 \quad(r \geq 0 \text { on } S) .
$$

It follows that on regular analytic super- $K_{0}$ surfaces the function $l(r)$ is a super- $K_{0}$ function and satisfies $l(r) \leq l_{0}(r) ; l(r)$ is a strictly super- $K_{0}$ function and satisfies the strict inequality for $r>0$ on $S$ if $S$ is not a $K_{0}$-surface. Also, on these surfaces we have $\phi_{1}^{\prime}(0)=0$, so that, since $\phi_{1}(r)$ is a super- $K_{0}$ function, for a given regular analytic super- $K_{0}$ surface and for a given pole $P$ on $S_{\text {, either } l}(r)$ is monotonic increasing on $S$ or there is an $r_{0}=r_{0}(S, P)>0$ such that $l(r)$ is monotonic increasing for $0 \leq r \leq r_{0}$ and monotonic decreasing for $r \geq r_{0}$ on $S$.

Again, we have

$$
\begin{gathered}
a(r)=a_{0}(r)+\phi_{2}(r), \\
a^{\prime}(r)=l_{0}(r)+\phi_{1}(r)=l(r),
\end{gathered}
$$

and

$$
\widetilde{\Xi}(r)=2 \pi+\widetilde{\circlearrowleft} \phi_{2}(r)
$$

On regular analytic super- $K_{0}$ surfaces we have

$$
\phi_{2}(r) \leq 0, \phi_{2}^{\prime \prime}(0)=0, \widetilde{\phi_{2}}(r) \leq 0 \quad(r \geq \text { on } S) .
$$

Hence on regular super- $K_{0}$ surfaces, $a(r)$ satisfies $a(r) \leq a_{0}(r)$; the strict inequality holds for $r>0$ on $S$ if $S$ is not a $K_{0}$-surface. Further, for a given regular analytic super- $K_{0}$ surface, and for a given pole $P$ on $S$, either $a(r)$ is a strictly sub- $K_{0}$ function, or there is an $r_{0}=r_{0}(S, P)>0$ such that $a(r)$ is a strictly sub- $K_{0}$ function for $0 \leq r \leq r_{0}$ and a strictly super- $K_{0}$ function for $r \geq r_{0}$ on $S$. The interval $0 \leq r \leq r_{0}$ on which $a(r)$ is a sub- $K_{0}$ function coincides with the interval on which $l(r)$ is increasing. 
From the properties of $\phi_{4}(r), \phi_{5}(r)$ and $\phi_{6}(r)$ described in Theorem 5.2, we deduce some inequalities of interest. Thus, on regular analytic super- $K_{0}$ surfaces we have the inequalities

$$
\begin{gathered}
a(r) \geq \frac{1}{4 \pi}\left[l_{0}(r) l(r)+K_{0} a_{0}^{2}(r)\right], \\
a(r) \geq \frac{1}{l_{0}^{\prime}(r)}\left[l_{0}(r) l(r)-2 \pi a_{0}(r)\right],
\end{gathered}
$$

and

$$
a(r) \geq a_{0}(r)-\frac{r}{2}\left[l_{0}(r)-l(r)\right],
$$

associated with $\phi_{4}(r), \phi_{5}(r)$, and $\phi_{6}(r)$ respectively, with the signs of $e$ quality holding for $r>0$ on $S$ if and only if $S$ is a $K_{0}$-surface.

When the proof in Theorem 4.6 is examined in light of the new basic inequalities for regular analytic super- $K_{0}$ surfaces, we find that $\phi_{7}(r)$ remains a monotonic, nondecreasing sub- $K_{0}$ function. The function $4 \pi \phi_{7}(r)$ is factorable in such a way that $\phi_{8}(r)$ is a factor; then, by Theorem 5.2, the other factor satisfies the inequality

$$
l(r)-l_{0}(r)-\sqrt{-K_{0}}\left[a(r)-a_{0}(r)\right] \leq 0 .
$$

Hence, using this last relation, on regular analytic super- $K_{0}$ surfaces we have the inequalities

$$
l(r) \leq l_{0}(r)-\sqrt{-K_{0}}\left[a_{0}(r)-a(r)\right] \leq l_{0}(r),
$$

and

$$
a_{0}(r) \geq a(r) \geq a_{0}(r)-\frac{1}{\sqrt{-K_{0}}}\left[l_{0}(r)-l(r)\right],
$$

with the signs of equality holding for $r>0$ on $S$ if and only if $S$ is a $K_{0}$-surface.

The $\phi$ functions related to geodesic circular sectors (see Theorem 5.1) have analogous properties on regular super- $K_{0}$ surfaces.

5.3. Surface characterization. Heretofore we have assumed $S$ to be either 
a sub- $K_{0}$ surface or a super- $K_{0}$ surface. In certain instances we have obtained, in the two cases, conclusions which are distinct except for the dividing class of $K_{0}$-surfaces. Thus by logical exclusion we obtain several characterizations of the indicated classes of surfaces.

For example, a regular analytic surface $S$ is a super- $K_{0}$ surface, but not a $K_{0}$-surface, if and only if for each pole $P$ on $S$ we have

$$
l(r)<l_{0}(r)
$$

for all $r>0$ on $S$.

Proof. In $\S 5.2$ we have shown that the condition $K \geq K_{0}, K \not \equiv K_{0}$, on $S$ implies (5.31). Conversely, if we should have $K_{1}<K_{0}$ at some $P_{1}$ on $S$, then we would have $K<K_{0}$ throughout some neighborhood of $P_{1}$, and therefore, in the neighborhood, we would have $l(r)>l_{0}(r)$; also, if we should have $K \equiv K_{0}$ on $S$, then we would have $l(r) \equiv l_{0}(r)$; hence (5.31) implies $K \geq K_{0}, K \not K_{0}$ on $S$.

In the same way we could establish similar results for each function in the following theorem.

THEOREм 5.3. The regular analytic surface $S$ is i) a sub- $K_{0}$ surface, but not a $K_{0}$-surface, ii ) a super- $K_{0}$ surface, but not a $K_{0}$-surface, or iii ) a $K_{0^{-}}$ surface, if and only if we have

$$
\begin{aligned}
\text { i) } \left.\left.\phi_{j}(r)>0, \quad \text { ii }\right) \phi_{j}(r)<0, \quad \text { or } \quad \text { iii }\right) \phi_{j}(r) \equiv 0, \\
\quad(j=1,2, \cdots, 6,8,9, \cdots, 15),
\end{aligned}
$$

respectively, for all poles $P$ and all $r>0$ on $S$.

By Theorem 1.2, it is evident that we might replace (5.32) with the differential conditions

$$
\begin{aligned}
\text { i } \left.) \subseteq \phi_{j}(r)>0, \quad \text { ii }\right) \subseteq \phi_{j}(r)<0, & \text { or } \quad \text { iii }) \subseteq \phi_{j}(r) \equiv 0 \\
& (j=1,2,4,5,6,8,13,14) .
\end{aligned}
$$

5.4. Geodesically similar curves. The preceding theory may be applied to more general configurations than geodesic circles and sectors. Thus we may study comparison functions which involve length and area functions relating to a class of curves upon an arbitrary surface $S$ as compared to the corresponding curves upon a $K_{0}$-surface or in the plane. 
It is evident that $r$ has heretofore played a dual role: it has served as the parameter for the family of geodesic circles (sectors) on $S$ with centers at the pole $P$, and it also has been a variable of the geodesic polar coordinate system. We now rephrase the previous conditions in terms of the parameter of the family of curves to be considered.

Condition $\mathrm{A}(k)$. For a given surface $S$ of nonpositive Gaussian curvature, and for a given one-parameter family of curves $C(k)$, a function $\lambda(k)$ of the parameter $k$ satisfies Condition A $(k)$ provided: $\lambda(0)=0$; for $k \geq 0, \lambda(k)$ is a continuous monotonic nondecreasing convex function of $k ; \lambda(k) \equiv 0$ if $S$ is a developable surface, but otherwise is monotonic increasing and strictly convex.

Condition $\mathrm{C}(k)$. For a given sub- $K_{0}$ surface $S$, and for a given oneparameter family of curves $C(k)$, a function $\tau(k)$ of the parameter $k$ satisfies Condition $\mathrm{C}(k)$ provided: $\tau(0)=0$; for $k \geq 0, \tau(k)$ is a continuous monotonic nondecreasing sub- $K_{0}$ function of $k ; \tau(k) \equiv 0$ if $S$ is a $K_{0}$-surface, but otherwise $\tau(k)$ is a strictly sub- $K_{0}$ function of $k$.

On a surface $S$ referred to geodesic polar coordinates $(r, \theta)$ with a given pole $P$, we first consider the family of curves $C(k)$ of parameter $k$ given by

$$
r=k f(\theta), k \geq 0,
$$

where $f(\theta)$ admits a continuous derivative and $f(\theta) \geq 1$. We remark that the condition $f(\theta) \geq 1$ is merely a normalization; for, if $f\left(\theta_{0}\right) \leq 1$ and $f(\theta) \neq 0$ in a closed interval, $\alpha \leq \theta \leq \beta$, then $f(\theta)$ is bounded away from zero in $(\alpha, \beta)$, say $f(\theta) \geq m>0$ in $(\alpha, \beta)$. Then a new parameter $k_{1}$ may be introduced by setting $k=m k_{1}$, so that

$$
r=k_{1} f_{1}(\theta)=k_{1}[m f(\theta)]
$$

and this representation satisfies our requirements. It may be noted that $f(\theta) \equiv 1$ presents the case of geodesic circles (sectors). The curves $C(k)$ of the family given by $(5.41)$ are said to be similarly situated or homothetic, and we shall call them geodesically similar.

THEOREM 5.4. Let $S$ be an analytic surface of nonpositive Gaussian curvature referred to geodesic polar coordinates with given pole $P_{0}$. Let $l_{P}(k ; \alpha, \beta)$ and $l_{S}(k ; \alpha, \beta)$ denote the lengths of the curve of the family $C(k)$ of (5.41) from $\theta=\alpha$ to $\theta=\beta,(\alpha<\beta)$, for the parameter value $k$ in the plane and on the surface $S$, respectively, and let $a_{P}(k ; \alpha, \beta)$ and $a_{S}(k ; \alpha, \beta)$ denote the 
areas of the sectors formed by the curve of the family $C(k), \theta=\alpha$, and $\theta=\beta$ $(\alpha<\beta)$, for the parameter value $k$ in the plane and on the surface $S$ respectively. Then the functions

$$
\lambda_{1}(k ; \alpha, \beta) \equiv l_{S}(k ; \alpha, \beta)-l_{P}(k ; \alpha, \beta)
$$

and

$$
\lambda_{2}(k ; \alpha, \beta) \equiv a_{S}(k ; \alpha, \beta)-a_{P}(k ; \alpha, \beta)
$$

satisfy Condition $A(k)$.

Proof (outlined). For $\theta_{0}$ fixed and $\alpha<\theta_{0}<\beta$, let

$$
\lambda_{0}\left(k, \theta_{0}\right) \equiv\left[\mu^{2}+\left(\frac{d r}{d \theta}\right)^{2}\right]^{1 / 2}-\left[r^{2}+\left(\frac{d r}{d \theta}\right)^{2}\right]^{1 / 2},
$$

where $r=r(\theta)$ is given by $(5.41)$. We find that $\lambda_{0}\left(0, \theta_{0}\right)=0$, and that $\partial \lambda_{0} / \partial k$ and $\partial^{2} \lambda_{0} / \partial k^{2}$ are nonnegative since $\mu\left(r, \theta_{0}\right)$ is a convex function of $r$. On verifying the other requirements, we have that, for each fixed value $\theta_{0}, \lambda_{0}\left(k, \theta_{0}\right)$ satisfies Condition $\mathrm{A}(k)$.

Since

$$
\lambda_{1}(k ; \alpha, \beta) \equiv \int_{a}^{\beta} \lambda_{0}(k, \theta) d \theta,
$$

it follows (See [4, Theorem 1, p. 287].) that $\lambda_{1}(k ; \alpha, \beta)$ satisfies Condition $\mathrm{A}(k)$. If the function $l_{S}(k ; \alpha, \beta)$ alone is considered, then the relations used also indicate that $l_{S}(k ; \alpha, \beta)$ is a convex function of $k$, that it is strictly convex if $S$ is not a developable surface, and that it is linear (as a function of $k$ ) if $S$ is developable.

Now, with (5.41),

$$
\lambda_{2}(k ; \alpha, \beta) \equiv \int_{\alpha}^{\beta} \int_{0}^{r}[\mu(\rho, \theta)-\rho] d \rho d \theta
$$

and its first and second derivatives are found to be nonnegative by use of the convexity of $\mu(r, \theta)$. The remainder of the argument is direct.

We find other results for the area functions: 
THEOREM 5.5. Let $S$ be an analytic sub- $K_{0}$ surface referred to geodesic polar coordinates with given pole $P_{0}$. Let $a_{S}(k ; \alpha, \beta), a_{S_{0}}(k ; \alpha, \beta)$, and $a_{P}(k ; \alpha, \beta)$ denote the areas of the sectors formed by the curve of the family $C(k)$ of (5.41) for the parameter value $k, \theta=\alpha$, and $\theta=\beta(\alpha<\beta)$ on the

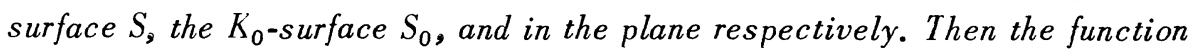

$$
\lambda_{2}(k ; \alpha, \beta) \equiv a_{S}(k ; \alpha, \beta)-a_{P}(k ; \alpha, \beta)
$$

is a monotonic nondecreasing sub- $K_{0}$ function of $k_{9}$ and the function

$$
\tau_{1}(k ; \alpha, \beta) \equiv a_{S}(k ; \alpha, \beta)-a_{S_{0}}(k ; \alpha, \beta)
$$

satisfies Condition $\mathrm{C}(k)$.

Proof. By Theorem 5.4, $\lambda_{2}(k ; \alpha, \beta)$ is nonnegative and monotonic nondecreasing. By calculation,

$$
\Im_{k} \lambda_{2}(k ; \alpha, \beta)=\int_{\alpha}^{\beta} \int_{0}^{r}\left[\Im_{\rho} \mu+\frac{\partial^{2} \mu}{\partial \rho^{2}}\left(f^{2}-1\right)-K_{0} \rho\right] d \rho d \theta>0
$$

for $r>0(k>0)$ on $S$ since $f(\theta) \geq 1$. Hence $\lambda_{2}(k ; \alpha, \beta)$ is a sub- $K_{0}$ function of $k$.

For the other function, we find that

$$
\frac{\partial \tau_{1}}{\partial k}=\int_{\alpha}^{\beta}\left[\mu(r, \theta)-\mu_{0}(r, \theta)\right] f(\theta) d \theta
$$

and

$$
\widetilde{\Xi}_{k} \tau_{1}(k ; \alpha, \beta)=\int_{\alpha}^{\beta} \int_{0}^{r}\left[f^{2} \Im_{\rho}\left(\mu-\mu_{0}\right)-K_{0}\left(\mu-\mu_{0}\right)\left(f^{2}-1\right)\right] d \rho d \theta .
$$

These are nonnegative by the proof of Theorem 3.3, and the rest of the argument is immediate.

5.5. The Steiner configuration. Let $C$ be an arbitrary closed convex curve in the plane, of length $L$ and area $F$, and let $C(\rho)$ be a curve parallel to $C$ at a distance $\rho$ from it, $\rho$ being measured along the outward normal to $C$, of length $L(\rho)$ and area $F(\rho)$. The family of curves $C(\rho)$ will be called a Steiner configuration; it is a classical result of Steiner $[2, \mathrm{p} .128]$ that 


$$
L(\rho)=L+2 \pi \rho
$$

and

$$
F(\rho)=F+\rho L+\pi \rho^{2}
$$

Generalizations of these formulas for curves lying on a curved surface have been given in $[1 ; 2]$, and explicit formulas found in the case of surfaces of constant curvature. We shall establish the sub- $K_{0}$ function property of some functions which involve the $L(\rho)$ and $F(\rho)$ functions for the Steiner configuration associated with a suitable curve $C$ on an arbitrary sub- $K_{0}$ surface, $K_{0}<0$. It is evident that our preceding theory for geodesic circles of center $P_{0}$ on $S$ is obtained from a Steiner configuration on $S_{*}$ if the curve $C$ is a geodesic circle of center $P_{0}$ on $S$.

Let the curve $C$ be a simple, closed, bounding, and differentiable curve on the surface $S$. Introduce a geodesic representation with coordinates $(u, v)$ in which $u=0$ is the curve $C$, and $v=$ constant are the geodesics orthogonal to $C$; further, let $v$ be the arc length of $C$ measured positively for motion on the curve which keeps the bounded area to the left, and let $u$ be the arc length of geodesics normal to $C$. Sufficient conditions for the validity of such a coordinate system in a region of $S$ have been given $[1 ; 2]$. We shall assume that our coordinate system is valid and term admissible those curves which satisfy the above conditions.

Then, for an admissible curve $C$ of length $L$ and area $F$, and for $\rho$ fixed, the length $L(\rho)$ of $C(\rho)$ is given by

$$
L(\rho)=\int_{C} \mu(\rho, v) d v,
$$

and the area $F(\rho)$ of $C(\rho)$ is given by

$$
F(\rho)=F+\int_{C} \int_{0}^{\rho} \mu(u, v) d u d v
$$

For a $K_{0}$-surface, $K_{0}<0$, Abascal [1, p. 843] has shown that these relations simplify to (in our notation)

$$
L_{0}(\rho)=L+l_{0}(\rho)-\frac{K_{0}}{2 \pi}\left[F l_{0}(\rho)+L a_{0}(\rho)\right]
$$




$$
F_{0}(\rho)=F+a_{0}(\rho)+\frac{L l_{0}(\rho)}{2 \pi}-\frac{K_{0} F a_{0}(\rho)}{2 \pi},
$$

where $l_{0}(\rho)$ and $a_{0}(\rho)$ are given in Lemma 2.4.

LEMmA 5.6. The functions $L_{0}(\rho)$ and $F_{0}(\rho)$ satisfy the relations

$$
\begin{gathered}
L_{0}^{\prime}(\rho)+K_{0} F_{0}(\rho)-2 \pi=0, \\
L_{0}^{\prime \prime}(\rho)+K_{0} L_{0}(\rho)=0,
\end{gathered}
$$

and

$$
\frac{L_{0}^{2}(\rho)}{4 \pi}+\frac{K_{0} F_{0}^{2}(\rho)}{4 \pi}-F_{0}(\rho)=M=\text { constant }
$$

for $\rho \geq 0$ on the $K_{0}$-surface $S_{0}$.

Proof. The first two relations follow easily from (5.53), (5.54), and the properties of $l_{0}(\rho)$ and $a_{0}(\rho)$. The third relation is immediate since the derivative of its left member is zero.

THEOREM 5.7. Let $S$ be an analytic sub- $K_{0}$ surface, and let $C(\rho)$ denote the curves of the Steiner configuration for an admissible curve $C$ on $S$. Then the length function $L(\rho)$ is a sub- $K_{0}$ function of $\rho ; L(\rho)$ is a strictly sub- $K_{0}$ function if $S$ is a strictly sub- $K_{0}$ surface, and it is a $K_{0}$-function of $\rho$ if $S$ is a $K_{0}$-surface. Further, the area function $F(\rho)$ is a strictly sub- $K_{0}$ function.

Proof. These properties of $L(\rho)$ were established in Theorem 2.2.

By calculation from (5.52) we get

$$
\widetilde{\Xi}_{\rho} F(\rho)=\int_{C} \int_{0}^{\rho} \widetilde{\Xi}_{u} \mu d u d v+\int_{C}\left(\frac{\partial \mu}{\partial u}\right)_{\rho=0} d v+K_{0} F .
$$

For our geodesic representation, it is known $[7, \mathrm{p} .188]$ that

$$
K_{g}(v)=\left[\frac{\partial \mu(u, v)}{\partial u}\right]_{u=0},
$$

where $K_{g}(v)$ is the geodesic curvature of $C$. By the Gauss-Bonnet theorem [7, p. 191$]$, noting that $C$ has no exterior angles, we get 


$$
\int_{C} K_{g}(v) d v=2 \pi-\iint_{F} K \mu d u d v \geq 2 \pi-K_{0} F .
$$

since $K \leq K_{0}$. With $\widetilde{\Xi}_{u} \mu \geq 0$ and (5.57), it follows from (5.56) that

$$
\Xi_{\rho} F(\rho) \equiv L^{\prime}(\rho)+K_{0} F(\rho) \geq 2 \pi,
$$

and then Theorem 1.2 ensures the result of the theorem.

We shall now make comparison between the length and area functions for Steiner configurations on a sub- $K_{0}$ surface $S$ and on the $K_{0}$-surface. However, our expressions may be considered to be functions formed with respect to $S$ alone because of (5.53), (5.54), and the known formulas for $l_{0}(\rho)$ and $a_{0}(\rho)$.

THEOREM 5.8. Let $S$ be an analytic sub-K $K_{0}$ surface, and let $C(\rho)$ denote the curves of the Steiner configuration for an admissible curve $C$ of length $L$ and area $F$ on $S$. Let $C_{0}(\rho)$ denote the curves of the Steiner configuration for any admissible curve $C_{0}$ of length $L_{0}=L$ and area $F_{0}=F$ on the $K_{0}$-surface $S_{0}$. Then the functions

$$
\tau_{2}(\rho) \equiv L(\rho)-L_{0}(\rho)
$$

and

$$
\tau_{3}(\rho) \equiv F(\rho)-F_{0}(\rho)
$$

satisfy Condition $\mathrm{C}(\rho)$, where $\rho$ is the parameter of the family.

Proof. There is equality in (5.57) if $S$ is a $K_{0}$-surface, and the proof using (5.51) and (5.52) is similar to those of Theorem 3.3 and Theorem 3.5.

Theorem 5.8 admits the corollary that the functions $L(\rho)$ and $F(\rho)$ satisfy the inequalities

$$
L(\rho) \geq L_{0}(\rho) \equiv L+l_{0}(\rho)+\frac{\left|K_{0}\right|}{2 \pi}\left[F l_{0}(\rho)+L a_{0}(\rho)\right]
$$

and

$$
F(\rho) \geq F_{0}(\rho) \equiv F+a_{0}(\rho)+\frac{L l_{0}(\rho)}{2 \pi}+\frac{\left|K_{0}\right| F a_{0}(\rho)}{2 \pi}
$$

both functions are strictly sub- $K_{0}$ functions and satisfy the strict inequalities 
for $\rho>0$ on $S$ if $S$ is not a $K_{0}$-surface, and they satisfy the equalities if $S$ is a $K_{0}$-surface. We remark that the conditions $L_{0}=L$ and $F_{0}=F$ were imposed to meet the requirements of Condition $\mathrm{C}(\rho)$. The sub- $K_{0}$ function properties and inequality relations above would hold equally well for any admissible $C_{0}$ such that $L_{0} \leq L$ and $F_{0} \leq F$.

We shall now establish some results for functions involving $L(\rho)$ and $F(\rho)$ which are analogous to the isoperimetric function and to its modifications.

THEOREM 5.9. Let $L(\rho)$ and $F(\rho)$ be the length and area functions, respectively, of the curves of a Steiner configuration on an analytic sub- $K_{0}$ surface $S$. Then the function

$$
\theta(\rho) \equiv \frac{L^{2}(\rho)}{4 \pi}-F(\rho)
$$

is a positive monotonic strictly increasing sub- $K_{0}$ function of $\rho$; further, if $C_{0}$ on the $K_{0}$-surface $S_{0}$ satisfies $L_{0}=L$ and $F_{0}=F$, then the function

$$
\tau_{4}(\rho) \equiv \frac{L^{2}(\rho)-L_{0}^{2}(\rho)}{4 \pi}-\left[F(\rho)-F_{0}(\rho)\right]
$$

satisfies Condition $\mathrm{C}(\rho)$.

Proof. It is known [6] that $\theta(\rho)>0$ on sub- $K_{0}$ surfaces. With (5.58) and Theorem 5.7, routine computations show that $\theta^{\prime}(\rho)$ and $ธ \theta(\rho)$ are positive, establishing the properties of $\theta(\rho)$. The properties of $\tau_{4}(\rho)$ are established in routine manner by the use of $(5.58)$ and Theorem 5.8.

Theorem 5.10. Let $L(\rho)$ and $F(\rho)$ be the length and area functions, respectively, of the curves of a Steiner configuration on a sub- $K_{0}$ surface $S$, and let $L_{0}(\rho)$ and $F_{0}(\rho)$ be the length and area functions, respectively, of the curves of a Steiner configuration on a $K_{0}$-surface $S_{0}$. Let the admissible curve $C_{0}$ on $S_{0}$ satisfy $L_{0}=L$ and $F_{0}=F$. Then the function

$$
\tau_{5}(\rho) \equiv\left[\frac{L^{2}(\rho)}{4 \pi}+\frac{K_{0} F^{2}(\rho)}{4 \pi}-F(\rho)\right]-\left[\frac{L_{0}^{2}(\rho)}{4 \pi}+\frac{K_{0} F_{0}^{2}(\rho)}{4 \pi}-F_{0}(\rho)\right]
$$

satisfies Condition $\mathrm{C}(\rho)$.

Proof. Obviously $\tau_{5}(0)=0$, and using (5.55) we get $\tau_{5}^{\prime}(\rho) \geq 0$ by (5.58). 
By another calculation we find that

$$
\begin{aligned}
& 4 \pi \circlearrowleft \tau_{5}(\rho) \geq\left\{2 L^{\prime}(\rho)\left[L^{\prime}(\rho)+K_{0} F(\rho)-2 \pi\right]+K_{0} L^{2}(\rho)+K_{0}^{2} F^{2}(\rho)\right. \\
& \left.-4 \pi K_{0} F(\rho)-4 \pi K_{0} M\right\}
\end{aligned}
$$

where the constant $M$ is given by $(5.55)$. We then use (5.58) just as we used (3.22) in the proof of Theorem 4.1, and we get

$$
4 \pi \circlearrowleft \tau_{5}(\rho) \geq\left[L^{\prime 2}(\rho)+K_{0} L^{2}(\rho)-4 \pi^{2}-4 \pi K_{0} M\right] \equiv h(\rho)
$$

where $h(\rho)$ is the function in brackets. By (5.53) and (5.54), we verify that

$$
4 \pi K_{0} M=L_{0}^{\cdot 2}(\rho)+K_{0} L_{0}^{2}(\rho)-4 \pi^{2},
$$

and when this is substituted in (5.59), it follows that $h(0) \geq 0$ since $L^{\prime}(0) \geq$ $L_{0}^{\prime}(0)$ by (5.58). By computation and use of Theorem 5.7 we find that $h^{\prime}(\rho) \geq 0$ for $\rho \geq 0$; hence $h(\rho) \geq 0$ for $\rho \geq 0$, and $\tau_{5}(\rho)$ is a sub-K $K_{0}$ function. Since further considerations show that the signs of equality hold above if and only if $S$ is a $K_{0}$-surface, we have, with the final remark that $\tau_{5}(\rho) \equiv 0$ if $S$ is a $K_{0^{-}}$surface, the result that $\tau_{5}(\rho)$ satisfies Condition $\mathrm{C}(\rho)$.

We remark that the last two theorems imply inequalities for the functions $L(\rho)$ and $F(\rho)$ somewhat similar to (4.13); we omit the formal statements.

Let the symbol $\Phi_{k}(\rho)$ denote the new functions produced from the functions $\phi_{k}(r)$ when the functions $r, a(r), a_{0}(r), l(r)$, and $l_{0}(r)$, associated with geodesic circles, are replaced by the functions $\rho, F(\rho), F_{0}(\rho), L(\rho)$, and $L_{0}(\rho)$, respectively, associated with the Steiner configuration of an admissible curve C. For example,

$$
\tau_{5}(\rho) \equiv \Phi_{3}(\rho)-M,
$$

where $M$ is the constant of (5.55). It may then be verified (indeed almost solely by inspection of the proof that the corresponding $\phi(r)$ function satisfies Condition $\mathrm{C}$ ) that the functions

$$
\Phi_{k}(\rho)-M \quad(k=4,5),
$$

and

$$
\Phi_{k}(\rho) \quad(k=6,7,8,9,10,11),
$$


satisfy Condition $\mathrm{C}(\rho)$.

Again, we might formulate a Condition $D(\rho)$ which is analogous to Condition D in the way that Condition C $(\rho)$ corresponds to Condition C. Then large parts of the theory in $\$ 3.3$ on "square-root" functions are found to apply to similar functions associated with a Steiner configuration. Finally, it may be shown that much of the theory in $\S \S 5.2,5.3$ can be generalized to hold for appropriate functions associated with Steiner configurations.

\section{REFERENCES}

1. E. V. Abascal, A generalization of Steiner's formulae, Bull. Amer. Math. Soc. 53 (1947), 841 - 844.

2. C. B. Allendoerfer, Steiner's formulae on a general $S^{n+1}$, Bull. Amer. Math. Soc. 54 (1948), $128-135$.

3. E. F. Beckenbach, Generalized convex functions, Bull. Amer. Math. Soc. 43 (1937), $363-371$.

4.

Some convexity properties of surfaces of negative curvature, Amer. Math. Monthly 55 (1948), $285-301$.

5. E. F. Beckenbach and R. H. Bing, On generalized convex functions, Trans. Amer. Math. Soc. 58 (1945), 220-230).

6. E. F. Beckenbach and T. Radó, Subharmonic functions and surfaces of negative curvature, Trans. Amer. Math. Soc. 35 (1933), 662-674.

7. L. P. Eisenhart, An introduction to differential geometry, Princeton, 1940.

8. E. L. Ince, Ordinary differential equations, New York, 1944.

9. M. M. Peixoto, Generalized convex functions and second order differential inequalities, Bull. Amer. Math. Soc. 55 (1949) 563-572.

10. H. Shniad, Convexity properties of means of moduli of analytic functions, Thesis, University of California, Los Angeles, 1948.

University of California, Los Angeles, and

State College of Washington 


\section{PACIFIC JOURNAL OF MATHEMATICS}

\section{EDITORS}

\section{R. U. FOEINSON}

University of California

Berkeley 4, California

E. HewitT

University of Washington

Seattle 5 , Washington
P. P. DILWOR TH

California Institute of Technology

Pasadena 4, California

E. F. BECKENBACH

University of California

Los Angeles 24, California

\section{ASSOCIATE EDITORS}

$\begin{array}{llll}\text { H. BUSEMANN } & \text { P. R. HALMOS } & \text { BØRGE JESSEN } & \text { J. J. STOKER } \\ \text { HERBERT FFDERER } & \text { IIEINZ HOPF } & \text { PAUL LÉVY } & \text { E. G. STRAUS } \\ \text { MARSHALL, IIALI } & \text { R. D. JAMES } & \text { GEORGE PÓLYA } & \text { KÖSAKU YOSIDA }\end{array}$

\section{SPONSORS}

UNIVERSITY OF BRITISH COLUMBIA

CALIFORNIA INSTITUTE OF TECHNOLOGY.

UNIVERSITY OF CAIJIFORNIA, BERKELEY

UNIVERSITY OF CAIJIFORNIA, DAVIS

UNIVERSITY OF CALIFORNIA, LOS ANGELES

UNIVERSITY OF CALIFORNI A, SANTA BARBARA

UNIVERSITY OF NEVADA

OREGON STATE COLLEGE

UNIVERSITY OF OREGON

\author{
UNIVERSITY OF SOU TIERN CALIFORNIA \\ STANFORD RESEARCH INSTITUTE \\ STANFORD UNIVERSITY \\ WASHINGTON STATE COLLEGE \\ UNIVERSITY OF WASHINGTON \\ AMERICAN MATHEMATICAL SOCIETY \\ NATIONAL BUREAU OF STANDARDS, \\ INSTITUTE FOR NUMERICAL ANALYSIS
}

Mathematical papers intended for publication in the Pacific Journal of Mathematics should be typewritten (double spaced), and the author should keep a complete copy. Manuscripts may be sent to any of the editors except Robinson, whose term expires with the completion of the present volume; they might also be sent to M.M. Schiffer, Stanford University, Stanford, California, who is succeeding Robinson. All other communications to the editors should be addressed to the managing editor, E. F. Beckenbach, at the address given above.

Authors are entitled to receive 100 free reprints of their published papers and may obtain additional copies at cost.

The Pacific Journal of Mathematics is published quarterly, in March, June, September, and December. The price per volume (4 numbers) is $\$ 8.00$; single issues, $\$ 2.50$. Special price to individual faculty members of supporting institutions and to individual members of the American Mathematical Society: $\$ 4.00$ per volume; single issues, $\$ 1.25$.

Subscriptions, orders for back numbers, and changes of address should be sent to the publishers, University of California Press, Berkeley 4, California.

Printed at Ann Arbor, Michigan. Entered as second class matter at the Post Office, Berkeley, California.

\section{UNIVERSITY OF CALIFORNIA PRESS • BERKELEY AND LOS ANGELES}




\section{Pacific Journal of Mathematics}

\section{Vol. 3, No. 2 \\ April, 1953}

William George Bade, An operational calculus for operators with spectrum

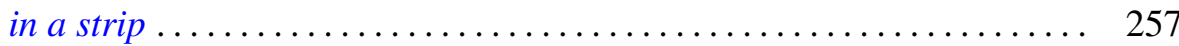

E. F. Beckenbach and Lloyd Kenneth Jackson, Subfunctions of several variables ..................................... 291

David Blackwell, Extension of a renewal theorem ................. 315

L. Carlitz, Some theorems on the Schur derivative ................ 321

Paul Arnold Clement, Generalized convexity and surfaces of negative curvature..................................... 333

Merrill M. Flood, On the Hitchcock distribution problem ............... 369

Watson Bryan Fulks, On the unique determination of solutions of the heat

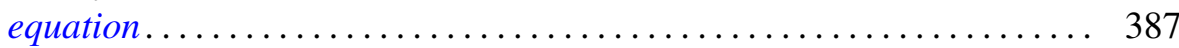

John W. Green, Length and area of a convex curve under affine transformation .................................... 393

William Gustin, An isoperimetric minimax .................. 403

Arthur Eugene Livingston, Some Hausdorff means which exhibit the Gibbs'

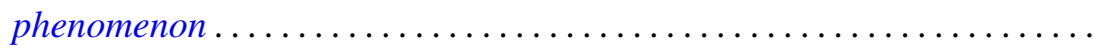

Charles Loewner, On generation of solutions of the biharmonic equation in the plane by conformal mappings ..................... 417

Gábor Szegő, Remark on the preceding paper of Charles Loewner ....... 437

Imanuel Marx and G. Piranian, Lipschitz functions of continuous

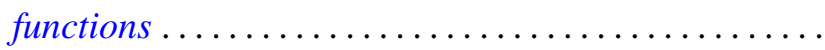

Ting-Kwan Pan, The spherical curvature of a hypersurface in Euclidean space ..

Ruth Lind Potter, On self-adjoint differential equations of second order ...

E. H. Rothe, A note on the Banach spaces of Calkin and Morrey...

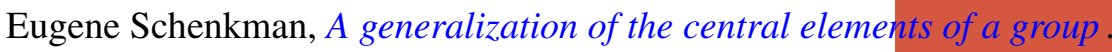

A. Seidenberg, A note on the dimension theory of rings .. . . 